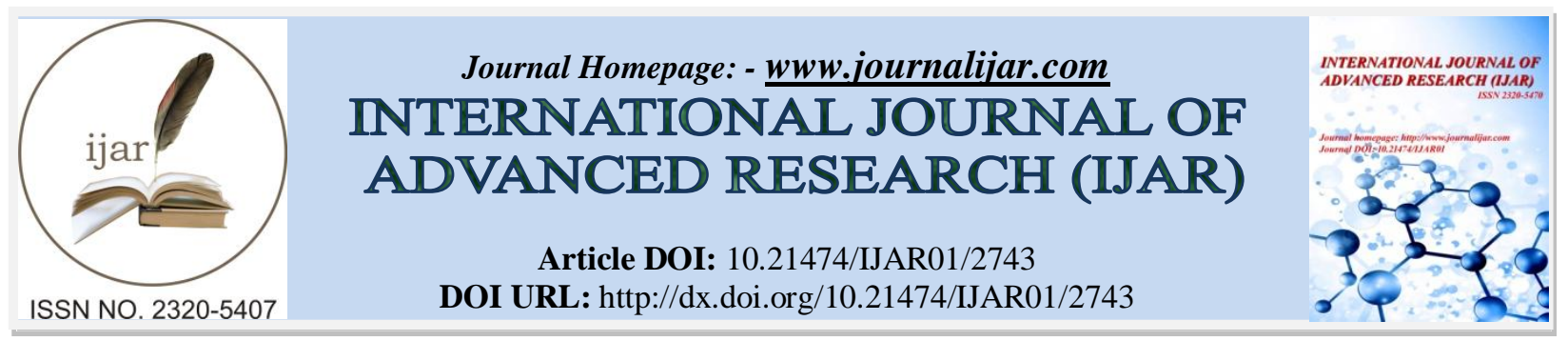

RESEARCH ARTICLE

\title{
PHILOSOPHIE : LA PEUR ET LE DESIR DE VENGEANCE A L'ORIGINE DE LA CONVERSION RELIGIEUSE.
}

Dr. David Pierre AVOCES. Université d'Abomey-Calavi (Bénin).

\section{Manuscript Info}

\section{Manuscript History}

Received: 15 November 2016

Final Accepted: 17 December 2016

Published: January 2017

Key words:-

Religious conversion - Reason -

Emotion - Fear - Desire - Vengeance -

Violence - Peace.

\begin{abstract}
The man uses the reason to distinguish the truly of the forgery. Yet, he is identified again like an emotional being. Besides, psychoanalysts and psychologists revealed that the emotion is the basis of the human existence. It releases by the fear and the desire of vengeance that push to the religious conversion. One knows that the fear is a violent fear facing a reality and that the desire is tension toward an object, source of satisfaction. In this sense, the role of the imagination is not to demonstrate anymore. Of the cruel gods to a merciful God, of the capricious gods to a God who frees, of idols to a God-mind, the convert leaves from these representations to encourage himself and to satisfy his desires.
\end{abstract}

Copy Right, IJAR, 2016,. All rights reserved.

\section{Introduction:-}

La religion est un effort pour maintenir la paix au sein de l'humanité. On sait qu'étymologiquement, le mot religion dérive du latin «religere » qui veut dire « respecter » et que par extension, il désigne le fait de vouer un culte. Dans le même ordre d'idées, il vient du verbe latin «religare» qui signifie «relier», pour exprimer le lien qui unit l'homme à Dieu le créateur ou à d'autres divinités, qu'il s'agisse de "religions du livre » ou de "religions de la coutume » pour reprendre les expressions d'Edmond Ortigues. De cette définition, il ressort que la conversion religieuse désigne l'adoption d'une nouvelle religion. Elle est souvent marquée par l'entrée dans un groupe de croyants et est accompagnée d'un acte symbolique comme par exemple, le baptême chez les Chrétiens, la profession de foi chez les Musulmans, l'initiation, suivie de la profession de foi dans les religions traditionnelles. Autrement dit, la conversion religieuse est le processus par lequel l'individu s'évertue à suivre une nouvelle voie en décidant d'abandonner les mauvaises pratiques ou les idées jugées fausses qu'il avait autrefois reçues. En se convertissant donc, l'individu passe par la foi pour rejoindre une communauté religieuse réputée dans la pratique et la transmission des valeurs préférées. Bien qu'étant une œuvre de la foi, même si quelquefois la raison peut intervenir dans les limites qu'on lui reconnaît, toutes les conversions religieuses se caractérisent par l'émotion. D'ailleurs, les recherches en psychologie et en psychanalyse ont révélé que l'émotion est la base même de la vie humaine. Elle est un phénomène psychologique qui pousse l'organisme à réagir face à une situation agréable ou désagréable.

Dans le présent article, nous voulons attirer l'attention du lecteur sur la peur et le désir de vengeance, deux émotions inconscientes et nocives pour la conversion religieuse. Il s'agit alors de mettre l'accent sur la connaissance et la maîtrise de soi pour que cesse la violence au sein des religions et que la paix soit une réalité, surtout dans les pays d'Afrique où le Boko Haram dicte sa loi. Car, la peur et le désir de vengeance suscitent chez les convertis, l'agressivité qui, à son tour, conduit à la violence ou à la guerre des religions. 


\section{A- La peur à l'origine de la conversion religieuse:- La peur des phénomènes naturels:-}

Dès son apparition dans le monde, l'homme a eu peur des phénomènes naturels. Alors, il s'est posé la question que se pose Leibniz dans Principes de la nature et de la grâce fondés en raison: Pourquoi il y a eu quelque chose plutôt que rien ? Pour Leibniz en effet, le rien serait plus facile à comprendre que quelque chose. C'est ce dernier qui met en œuvre la raison pour aider l'homme d'une manière ou d'une autre à comprendre et à expliquer tout ce qui existe. C'est alors qu'il aurait compris que les phénomènes naturels ont leur cause dans l'existence d'une autre chose dont ils sont l'effet. On comprend dès lors qu'à ses débuts, l'humanité ne pouvait pas conceptualiser directement et rationnellement l'idée de Dieu. Cela veut dire qu'en fait, la peur des phénomènes naturels serait à l'origine de la conception de Dieu et par conséquent, aurait conduit à la conversion religieuse. Ainsi, au départ, les hommes savaient qu'il y avait une vie et qu'ils en faisaient partie ; mais ils ne comprenaient pas les phénomènes naturels. C'est donc la peur de ces derniers qui poussait spontanément les premiers hommes à l'animisme. Ces phénomènes qu'ils ne comprenaient pas étaient entre autres : le soleil, le vent, les eaux, les plantes, les animaux, le feu, la foudre, les éruptions volcaniques, le tonnerre etc.

En marge de ces phénomènes, nous pouvons parler de la mort qui constitue pour l'homme une source d'angoisse. La peur liée à la mort provient de l'événement lui-même et des conséquences qui peuvent en découler. Pour un individu qui meurt, on sent la souffrance et la solitude chez les membres de sa famille. Après la mort, la question se pose encore de savoir si l'individu peut jouir d'une vie éternelle, en allant au Paradis. De plus, chacun se demande si après sa mort, quelqu'un pourrait s'occuper de sa famille et de ses biens. Blaise Pascal l'a bien compris quand il montre dans son œuvre Pensées, la peur qui nous anime au sujet de la mort. Dans le monde, tout se passe comme si les hommes sont enchaînés et condamnés à la mort. Certains étant chaque jour égorgés à la vue des autres, ceux qui restent, c'est-à-dire les vivants, voient leur propre condition dans celle de leurs semblables. En attendant leur tour, ils ont peur et se regardent avec douleur et sans espérance. S'inspirant de cette image de la condition humaine, Freud nous apprend la même chose quand, parlant de la mort des hommes, il affirme : «Leur mort fait naître la conscience douloureuse. ${ }^{1}$ De ces points de vue, la mort apparaît comme un phénomène qui pousse à réfléchir, en ce sens qu'elle conduit à la conversion religieuse à travers la peur qu'elle suscite.

Cela se comprend dans la mesure où l'homme est le seul animal qui sache qu'il doit mourir. De tous les êtres vivants, il possède de manière privilégiée la conscience ; une faculté qui le renseigne sur sa propre mort à partir de la mort d'autrui. Ainsi, il se plaint toujours d'avoir été créé mortel. C'est pourquoi, pour notre part, il ne serait pas exagéré de dire que l'homme est devenu religieux quand il a commencé par enterrer ses morts. Toutefois, il faut reconnaître que la mort ne doit pas être une source de peur, en ce sens qu'elle est un phénomène biologique. Elle n'est donc ni un accident, ni une réalité contingente ; mais une partie intégrante du système vivant. Dans La logique $d u$ vivant, François Jacob fait d'ailleurs remarquer que la mort est inhérente au monde animal et à son évolution. Elle est une nécessité inéluctable et une exigence de la vie.

La même idée se retrouve chez Heidegger qui, dans Introduction à la métaphysique soutient que l'homme est un être-pour-la-mort. Si tel est le cas, il serait stérile et vain de passer le temps à penser à notre finitude. L'essentiel pour le converti et pour tout homme est de bien agir pour améliorer ses conditions de vie. En fait, la saisie de notre finitude et de notre mort n'aura de sens que si elle nous pousse à de bonnes actions. Car, s'il est vrai que la mort est considérée comme la destruction du corps, il faut néanmoins reconnaître que quelque chose résiste à cette destruction. Il s'agit des œuvres que nous avons accomplies au cours de notre existence. C'est là notre revanche sur la mort et en même temps, l'espoir de toutes les victimes de la méchanceté humaine. Même si la vie ici-bas est éphémère, le fait de la vivre est à notre avis, un fait éternel.

Par ailleurs, ne pouvant pas comprendre pourquoi la nature pouvait se déchaîner dans des orages, des ouragans, des sécheresses et des inondations qui pouvaient tout détruire, l'homme est obligé de chercher une protection auprès de l'être suprême. En outre, dans une existence harassante où l'homme est confronté à des difficultés, la recherche de Dieu serait pour lui la solution adéquate. A l'instar de l'homme « primitif», l'homme des sociétés modernes croit en Dieu pour lutter contre la peur des phénomènes naturels et pour obtenir un bon niveau de vie. D'ailleurs, le fait d'observer la nature, de voir le jour, la nuit et les saisons se succéder ; de voir les fleurs s'épanouir périodiquement et les plantes se débarrasser de vieilles feuilles pour porter de nouvelles a amené les hommes à concevoir une force animée ; mieux, un esprit dans chacun des phénomènes de la nature. On comprend dès lors que c'est la peur des

\footnotetext{
${ }^{1}$ Sigmund FREUD, Essai de psychanalyse, Paris, Payot, 1972, p.215
} 
phénomènes naturels qui a poussé les hommes à imaginer un dieu de la pluie, de la terre, des cieux, du soleil, de l'air, du feu, de l'eau, etc.

Contrairement à ce que nous dit Placide Tempels dans La philosophie bantoue, la raison n'est pas à l'origine de la conversion religieuse. Pour notre part, la peur des phénomènes naturels pousse les hommes à se tourner vers Dieu pour l'adorer. Il s'agit là d'une réalité qui ne laisse aucun peuple indifférent. Qu'il s'agisse de «peuples civilisés » ou «non-civilisés», les réalités sont les mêmes. C'est pourquoi, bien que Raoul Allier ait montré que la conversion religieuse relève de l'émotion, nous ne sommes pas d'accord quand il nous parle seulement des «peuples indigènes », des «peuples primitifs » ou des «peuples non-civilisés ».

Dans le paganisme comme dans les religions révélées, la peur des phénomènes naturels pousse les hommes à penser qu'ils peuvent influencer sur les humeurs des dieux, des esprits ou des génies afin de leur plaire pour qu'ils deviennent favorables. Dans le même ordre d'idées, ils pensent que ces dieux, ces esprits et ces génies doivent agir selon leurs humeurs et leurs caprices. Ils ont très tôt compris qu'il fallait les écouter et les respecter pour se faire exaucer et pour se faire libérer de la peur des phénomènes naturels. Nous pouvons dès lors comprendre l'apparition des rituels de toutes sortes pour invoquer les puissances célestes. Par les rituelles, les hommes, de toute société, de toute langue et de toute culture apaisent les phénomènes naturels, en honorant les dieux et en les amenant à satisfaire à leur demande. On comprend alors le sens des rituels de naissance, d'initiation, de réconciliation, de protection, de mort qui s'observent à travers toutes les religions.

\section{La peur des malédictions ancestrales:-}

Dans les sociétés africaines modernes, la conversion religieuse se justifie de plus en plus par la peur des malédictions ancestrales. En effet, pour la plupart des personnes qui se convertissent, les ancêtres sont désormais identifiés comme la source de toutes malédictions, ou de toutes difficultés. Autrement dit, pour ceux qui se convertissent, il faut rompre le lien avec les ancêtres pour échapper aux difficultés de l'existence. De ce point de vue, le converti se fait une conception persécutive du mal qui se transmet à travers les liens de sang et à travers les générations. Les nombreuses conversions enregistrées depuis plusieurs années dans les religions monothéistes comme le Christianisme et l'Islam s'expliquent par la peur des malédictions ancestrales. Les difficultés de l'existence étant souvent attribuées à la sorcellerie ou aux esprits ancestraux, l'individu se convertit pour rompre avec le passé.

Dans les sociétés traditionnelles, pour échapper à l'échec, à la maladie et à la mort, il faut fréquemment apaiser les puissances persécutives en choisissant les rites propitiatoires les plus appropriés afin de resserrer les liens avec les ancêtres du lignage et les ascendants immédiats. Mais dans les religions du livre (L'Islam et le Christianisme), les personnes converties rompent le lien avec les esprits ancestraux pour se libérer de la peur des malédictions ancestrales et se laisser conduire par le Dieu créateur. C'est justement ce que nous apprend le couple Ortigues par l'affirmation suivante : «Dans l'islamisme ou le christianisme on exorcise, on expulse la puissance du mal qui vous possède. $»^{2}$

Il s'agit le plus souvent d'une rupture qui pousse à briser les liens familiaux. Car le converti pense que le démon œuvre à travers les liens de sang et que le Dieu créateur du ciel et de la terre est capable de rompre ces liens pour lui permettre de jouir du bonheur à travers sa bénédiction. Pour lui, la conversion garantit la protection contre le pouvoir maléfique des ancêtres. Depuis quelques décennies, la multiplication des Eglises Evangéliques et Pentecôtistes sur le continent africain donne la preuve de l'intention de rompre avec le lien ancestrale chez la plupart des personnes converties. De tels exemples nous donnent la preuve que la conversion religieuse en Afrique relève de l'émotion comme le souligne Raoul Allier dans La psychologie de la conversion chez les peuples non-civilisés. De plus en plus, on constate que les pratiques relatives aux religions traditionnelles sont abandonnées au profit de celles qui s'observent dans les religions monothéistes. Il n'est pas rare de voir les Eglises Evangéliques et Pentecôtistes lutter jour et nuit contre la sorcellerie, le démon et surtout contre les malédictions ancestrales ; car les génies, les esprits des morts anciens et nouveaux qui peuplent les maisons, les forêts, les rivières, les arbres sont désormais identifiés comme les causes de tout blocage chez l'individu qui aspire à la conversion religieuse.

${ }^{2}$ M.C.- E. ORTIGUES, Edipe africain, Paris, L’Harmattan, 1966, p. 129 
Précisons avec intérêt que la peur des malédictions ancestrales et le désir de rompre les liens avec les ancêtres s'expliquent par le fait que les conséquences des péchés commis par ces derniers retombent selon les convertis, sur les générations présentes et futures. Les effets de la malédiction ancestrale sur une personne se traduisent par des maladies chroniques, des psychopathologies (névroses et psychoses), des excès émotifs, des pertes financières répétées, une succession de morts non naturelles dans l'entourage de la personne, des troubles de comportement, des problèmes de stérilité et d'impuissance sexuel, des divorces répétés, des excès de colère etc.

Ces aléas de la vie quotidienne sont souvent interprétés comme des conséquences ou comme des effets de la malédiction ancestrale. Autrement dit, ils sont considérés comme les plans de l'ennemi ou du diable pour éprouver les individus et les détourner du salut de Dieu. C'est pourquoi, dans Sorcellerie et prière de délivrance, Meinrad Hebga nous donne son point de vue sur la question du rapport entre l'individu et les ancêtres : "De toute façon, les mânes des ancêtres ont chez nous un statut spécial, unique; nous leur vouons un culte religieux si important que la religion traditionnelle africaine a pu être appelée parfois ancestralisme. Mais un théologien comme Boladji Idowu, d'Ibadan, conteste une telle appellation. Selon lui, c'est finalement au dieu suprême que l'on offre sacrifice et libations. ${ }^{3}$

Les différents blocages de la vie des chrétiens ou des musulmans sont attribués à une maladie spirituelle qui affecte directement ou indirectement leur relation à Dieu. Car, bien qu'étant convertis, les fidèles peuvent encore être poursuivis et tourmentés par les esprits ancestraux. Mais précisons au passage que tous les esprits ancestraux ne poursuivent pas et ne tourmentent pas. Chez les Mossi comme chez beaucoup d'Africains, un ancêtre bienveillant peut apparaître dans le rêve afin de prévenir d'un danger imminent. Cela veut dire que les ancêtres peuvent se révéler bienveillants ou persécuteurs, selon les circonstances. A ce sujet, voici encore ce que nous apprend Hebga : «Ne devraient être regardés comme bons et bienveillants que ceux qui, véritables anges tutélaires nous témoigneraient leur sollicitude sans nous réduire en esclavage, et sans nous imposer des conditions qui blessent notre conscience morale et notre foi religieuse. ${ }^{4}$

\section{La peur des ennemis:-}

Une autre raison qui conduit à la conversion religieuse est la peur des ennemis. Pour la plupart des personnes converties, l'ennemi représente un individu asocial et mauvais, en ce sens qu'il veut le mal des autres. Autrement dit, l'ennemi est un persécuteur qui fait peur au converti et à sa famille. Sa seule présence peut être considérée comme une source de peur et de blocage. Il s'agit de la peur psychologique et de blocage dans la réussite des affaires : maladies, dépérissement, réduction de l'énergie vitale, échec, stérilité, mort etc. qui sont attribués à l'action malveillante des ennemis, surtout dans les sociétés africaines.

D'ailleurs dans Edipe africain, Marie-Cécile et Edmond Ortigues ont montré que ces maux et d'autres encore sont souvent l'objet des délires de persécution dans les sociétés africaines. Ils nous donnent l'exemple de Fari, un jeune lycéen originaire de Dakar. Fari est un musulman et candidat au baccalauréat technique. Il souffre d'insomnie et de céphalées violentes. Il faisait les pires rêves qui le poussaient à la folie. Son père avait décidé d'inscrire à l'école un de ses cadets. Mais puisque ce dernier était trop jeune et que Fari était plus âgé, il est entré à l'école à la place du cadet. Pour Fari en effet, son frère cadet serait à l'origine de sa maladie, en ce sens qu'il lui avait ravi la position qui devait être la sienne. Alors, la réussite scolaire pour Fari consiste à tuer son frère cadet. Ainsi, il ne souffrirait plus d'aucune maladie et il réussirait brillamment ses études. Dans le cas d'espèce, le sentiment de culpabilité est externe et l'agressivité s'exprime principalement sous la forme de réactions persécutives. Dans les cultures africaines, les rapports interpersonnels sont colorés par le fait que chacun se perçoit facilement comme persécuté par l'autre. Cela se comprend dans la mesure où l'individu qui souffre d'un mal, accuse le plus souvent un ennemi au sein ou en dehors de sa famille. Il est donc trop facile d'attribuer à l'action de l'ennemi, tous les maux dont on souffre.

A partir de ce constat, il serait intéressant de relever au passage une analogie entre la conversion dans les religions traditionnelles et celle qui s'observe dans les religions monothéistes. Dans La philosophie bantoue, Placide Tempels

\footnotetext{
${ }^{3}$ Meinrad HEBGA, Sorcellerie et prière de délivrance : réflexion sur une expérience, Paris, Présence

Africaine, 1982, p. 134

${ }^{4}$ Ibidem, pp. 136 - 137
} 
a fait remarquer que la peur de l'ennemi fait retourner les Bantous superficiellement convertis au Christianisme, à la religion de leurs ancêtres. Car dans les sociétés africaines traditionnelles, l'ennemi est le plus souvent considéré comme l'auteur du malheur ou plus précisément celui qui réduit l'énergie vitale, en ce sens qu'il est capable de conduire à la mort. La même conception se retrouve chez les personnes converties au monothéisme (Christianisme et Islam). Pour ces derniers en effet, la conversion religieuse apparaît comme la solution à la peur de l'ennemi. Nombreuses sont d'ailleurs les chansons religieuses qui véhiculent une telle idée. Dieu apparait dès lors comme le vainqueur de l'ennemi. Quiconque se convertit au Christianisme ou à l'Islam n'a plus rien à craindre.

Dans le psychisme des personnes converties aux religions bibliques, tout se passe comme si l'ennemi n'était à leurs yeux qu'un monstre mythique qu'il faut nécessairement abattre pour avoir la paix. Son action destructrice a, selon eux, une nature spirituelle. Pourtant, dans les Evangiles, le Seigneur Jésus nous invite à étendre son commandement d'amour jusqu'aux ennemis. Dans 1'Evangile selon Saint Matthieu, nous avons les propos suivants : "Vous avez entendu qu'il a été dit : tu aimeras ton prochain et tu häras ton ennemi. Eh bien! Moi je vous dis : aimez vos ennemis et priez pour vos persécuteurs, afin de devenir fils de votre Père qui est aux cieux, car il fait lever son soleil sur les méchants et sur les bons, et tomber la pluie sur les justes et les injustes. Car si vous aimez ceux qui vous aiment, quelle récompense aurez-vous? Les publicains eux-mêmes n'en font-ils pas autant? Et si vous réservez vos saluts à vos frères, que faites vous d'extraordinaire? Les païens eux-mêmes n'en font-ils pas autant? Vous donc, vous serez parfaits comme votre Père céleste est parfait. ${ }^{5}$

En fait, Jésus nous donne comme leçon d'aimer le pécheur ou l'ennemi, tout en détestant le péché. Autrement dit, nous devons agir comme un médecin qui combat la maladie en aimant le malade qui a besoin de guérison. En d'autres termes, nous devons lutter contre le mal que commettent nos ennemis tout en manifestant à leur égard, une immense charité. On peut dans cet ordre d'idées se référer au conseil de l'apôtre Paul dans l'Epître aux Romains : si nous aimons nos ennemis, nous serons vainqueurs du mal par le bien.

\section{La peur des punitions ici-bas:-}

Dans l'Evangile selon Saint Matthieu, Jésus nous met en garde contre la recherche effrénée du bonheur et du bienêtre à travers les moyens non recommandables : "Cherchez avant tout le royaume de Dieu et sa justice, et le reste vous sera accordé par surcroît. ${ }^{6}$ En s'inspirant de ce propos de Jésus, saint Paul, dans la Deuxième Epître aux Corinthiens souligne que la recherche du bonheur, sous toutes ses formes ne doit pas être la première préoccupation du converti ou de l'homme en situation dans le monde. L'essentiel est de connaître Dieu, de l'adorer et de faire sa volonté. C'est pourquoi, pour l'apôtre Paul en effet, la souffrance doit être acceptée par le converti, comme une participation à celle de Jésus. En d'autres termes, le converti doit être capable de boire avec Jésus, à la coupe d'amertume. Il doit être capable de souffrir et de mourir avec Jésus pour ainsi entrer avec lui dans son royaume. De ce point de vue, la souffrance ne doit plus être vue comme une punition ici-bas. Cela veut dire qu'en réalité, le converti ne doit plus avoir peur de quoi que ce soit. Tout ce qu'il vit en bien ou en mal doit être vécu en Dieu, pour Dieu et avec Dieu.

A partir de cette considération, une question pourrait venir à l'esprit du lecteur averti : doit-on accepter la souffrance sans rien faire pour y échapper? A notre avis, la réponse à cette question doit être négative. Pour notre part, Dieu n'est pas injuste et ne cautionne jamais l'injustice. De plus, il n'a jamais voulu le mal, mais toujours le bien de l'humanité. C'est d'ailleurs pourquoi il nous a dotés des facultés intellectuelles telles que : la conscience, la volonté, la liberté, la responsabilité, la raison, l'intelligence, la mémoire, l'imagination, pour ne citer que celles-là, afin de bien penser pour bien agir en faisant des choix libres et responsables pour notre bonheur et pour notre bien- être. Cela veut dire qu'en réalité, notre bonheur peut dépendre de nous-mêmes, si nous œuvrons vraiment dans ce sens et si nous agissons de façon responsable dans la société. Restons dans la même logique pour dire que le malheur peut dépendre de nous si nous n'exerçons pas bien nos facultés intellectuelles pour opérer des choix en vue d'une existence heureuse.

En résumé, si le bonheur peut dépendre de nos choix, le malheur peut en dépendre aussi. Nous n'ignorons certes pas la question du déterminisme, mais nous voulons souligner avec rigueur qu'il est inutile que les convertis se réfugient dans les églises, dans les mosquées et dans les religions traditionnelles pour échapper aux punitions ici-bas. En fait, Dieu reste égal à lui-même et n'est jamais responsable du mal de l'homme. Tant que l'homme ne changera pas de mentalité et de comportement ; tant qu'il ne va pas travailler avec une bonne conscience pour améliorer sa condition

\footnotetext{
${ }^{5}$ Mt 5, 43- 48

${ }^{6}$ Mt 6, $33-34$
} 
de vie et celle des autres, il ne peut échapper aux punitions ici-bas. Cela signifie qu'en réalité, Dieu ne met pas fin aux punitions ou à la souffrance de l'homme après son adhésion à une religion. Ce qui importe pour Dieu et qui passe par-dessus tout, c'est la recherche de son royaume et de sa justice à travers le respect des commandements. Si cela est compris et bien compris, nous pouvons prétendre échapper aux punitions ici-bas. Toutes les personnes converties qui n'auront pas compris cette leçon prendront la vie comme un fardeau dont il faut se débarrasser en passant d'une religion à une autre ou de certaines religions à d'autres.

Ainsi, à l'instar du tradithérapeute qui soumet son client à de nombreux interdits, Dieu lui aussi nous soumet à des interdits. Chez le tradithérapeute, enfreindre les interdits, c'est s'exposer à ne pas guérir du mal dont on souffre et de mourir par conséquent, de sa maladie. Dans la même logique, chez le croyant, l'ataraxie (absence de trouble ou tranquillité de l'âme) comporte elle aussi des exigences, c'est-à-dire des interdits qui se retrouvent dans les commandements de Dieu, du moins, sous leur forme prohibitive. Par exemple, si le converti comprend qu'il doit aimer son prochain comme lui-même, il peut éviter la violence et les conflits sociaux en favorisant ainsi la paix en lui et autour de lui. S'il comprend qu'il ne doit pas corrompre ou se faire corrompre, qu'il ne doit pas voler ou détourner le bien public, il doit travailler avec une bonne conscience pour satisfaire ses besoins fondamentaux (se nourrir, se soigner, s'instruire, se loger, et se vêtir), pour rendre service à la société et pour développer son pays. Cela ferait d'ailleurs de lui un patriote et un modèle à suivre. S'il sait qu'il doit s'occuper de ses enfants et bien les éduquer, il aura une famille heureuse, épanouie et contribuera ainsi à la formation de bons citoyens pour son pays. En agissant de la sorte, le converti n'aura plus peur des punitions ici-bas. Il saura que pour lui, le Paradis de Dieu commence déjà ici-bas, parce qu'il est devenu un bon converti. Dans ce sens, nous pouvons nous référer à cette formule de Ludwig Feuerbach : «Exister veut dire être sujet. » ${ }^{7}$

Dans le même ordre d'idées, nous pouvons nous référer à ce que nous dit Lucrèce (philosophe latin, 98 à 55 avant Jésus Christ), dans De la nature : si, en sentant sur leur cœur le poids qui les accable, les hommes pouvaient aussi connaître l'origine de leur mal et d'où vient leur lourd fardeau de misère, ils ne vivraient pas comme ils vivent trop souvent, en ignorant ce qu'ils veulent, cherchant toujours une place nouvelle comme pour se libérer de leur charge.

\section{La peur des punitions dans l'au-delà:-}

Jusqu'ici, nous avons constaté que toute religion propose à ses adeptes, le système de la carotte et du bâton : punition divine, en cas de mauvaises actions et récompense, en cas de bonnes actions. En habituant les hommes à une telle logique il est clair que leur conversion religieuse ne peut relever que de l'émotion et non de la raison. Plus on médite cette idée, plus on en sent la vérité. Plus on la conteste, plus on donne l'occasion de l'établir encore par de nouvelles preuves.

En effet, dans les religions traditionnelles, le rapport existant entre la morale, la religion et la manière dont les jugements se font sur terre amènent les hommes à se faire une idée du jugement dans l'au-delà. En d'autres termes, l'homme se fait une conception de l'autre vie, c'est-à-dire de l'au-delà qu'il se représente généralement comme une continuation de la vie terrestre. Dans l'imaginaire collectif, le pays des morts est fort semblable au pays des vivants, en ce sens que l'on pense que les bons seront récompensés, tandis que les mauvais seront punis ou châtiés, exactement comme cela se fait dans le monde ici-bas. On pense que dans l'au-delà, se trouvent les mêmes habitudes, les mêmes usages, le même genre de vie. Dès lors, le traitement réservé à ceux qui ne sont plus de ce monde et qui vont dans l'au-delà dépend du genre de vie qu'ils ont mené sur cette terre, c'est-à-dire dans ce monde. Autrement dit, selon la logique humaine, le jugement et le traitement réservés à l'individu dans l'au-delà relèvent du rang qu'il occupait dans sa famille, dans sa tribu. Cela dépend aussi de sa situation sociale, de sa richesse, de sa puissance magique, de son intelligence, de sa vigueur, de sa capacité à faire le bien ou le mal. La même idée est exposée dans La psychologie de la conversion chez les peuples non-civilisés où Raoul Allier montre que, selon la logique des «peuples primitifs» ou «peuples indigènes», le roi demeure roi, au pays des morts, l'esclave demeure esclave, celui qui a été malade ou malheureux voit se poursuivre sa maladie et sa misère, tandis que le bonheur suit au-delà du tombeau, l'homme qui en a joui sur cette terre. A notre avis, la peur des punitions dans l'au-delà serait encore à l'origine de la conversion religieuse.

${ }^{7}$ Ludwig FEUERBACH, Principes de la Philosophie de l'avenir, Paris, P.U.F., 1842, p. 220 
Dans les religions traditionnelles par exemple, on pense que les dieux châtient les malfaiteurs qui ont agi sur terre contre leurs serviteurs dévoués ou contre leurs adorateurs fidèles. Autrement dit, les dieux doivent punir dans l'audelà, ceux qui agissent mal sur terre après leur mort. De ce point de vue, les dieux qui habitent le pays des morts apparaissent dans les religions traditionnelles comme des juges qui étendent leur juridiction sur tous les actes des hommes, qu'ils agissent en bien ou en mal. Mentionnons avec intérêt que dans les religions traditionnelles, les dieux n'ont pas seulement pour rôle de juger les morts et de les châtier. Ils sont aussi pourvoyeurs de récompenses. Ils sont considérés encore comme des réparateurs des injustices commises dans le monde ici-bas. Car, on pense que ceux qui subissent l'injustice de la part des ennemis ou des plus forts de ce monde sont aussi consolés.

S'agissant des religions révélées (l'Islam et le Christianisme), la peur des punitions dans l'au-delà ou plus précisément, la peur de l'Enfer serait aussi l'une des raisons pouvant pousser les individus à se convertir. D'ailleurs, le discours sur la peur de l'Enfer est assez présent dans l'histoire des religions monothéistes. Tout porte donc à croire que ceux qui se convertissent craignent le jugement de Dieu et l'entrée en Enfer pour se conduire droitement. La figure de l'Enfer est bien l'image de la sanction des fautes. Les Saintes Ecritures, témoignent que Dieu jette dans l'Enfer ceux qui, sur cette terre, se sont éloignés de la justice pour choisir les voies tortueuses du mal. On se rappelle à juste titre du sermon de Jésus sur la montagne exposé dans l'Evangile selon saint Matthieu. Ici, Jésus nous parle de la géhenne de feu qui détruira le malfaiteur qui aura librement choisi de mener une existence en désaccord avec la loi de Dieu.

La même idée se retrouve dans le Coran : "Ceux qui font le mal et qui se font cerner par leurs péchés, ceux là sont les gens du feu où ils demeureront

éternellement. ${ }^{8}$ Une telle idée entraîne chez l'homme, la peur des sanctions dans l'au-delà. Dans le même sens, elle amène l'homme le plus mauvais à se convertir pour chercher refuge dans la foi en Dieu, dans l'espoir d'être sauvé de ses fautes. Cela veut dire qu'en réalité, dans les religions monothéistes, la peur de l'Enfer tient en respect un nombre important de personnes converties. En craignant le jugement de Dieu et l'Enfer après la mort, le converti est obligé de vivre selon les préceptes de la foi ; car, il sait que là haut, c'est-à-dire dans l'au-delà, les comptes seront faits et que son âme sera jugée si, ici-bas, il s'est livré au péché.

L'idée de la peur de l'Enfer est encore développée dans Gorgias de Platon où Socrate tente longuement de faire entendre à Calliclès qu'il vaut mieux être tempérant et intègre ici-bas, au lieu de prendre le parti de l'intempérance et de l'immoralisme. Mais, puisque Calliclès ne voulait pas entendre la voix de la raison, Socrate tire sa dernière cartouche et emploie un langage religieux en tenant un discours menaçant relatif à la sanction des âmes après la mort. Pour Socrate en effet, la peur de l'Enfer doit nous obliger à bien agir ici-bas.

Mais, mentionnons que pour le converti, la peur de l'Enfer n'est qu'une erreur en ce sens qu'elle relève de l'imagination. Déjà, dans l'antiquité, des voix se sont élevées contre la religion, notamment dans l'épicurisme. En effet, si beaucoup de religions pensent détenir la vérité au sujet de la peur de l'Enfer qui pousse le converti à vivre selon les règles morales ici-bas, afin d'entrer dans le Paradis, tel n'est pas le cas chez Epicure qui nie totalement l'existence d'un au-delà après la mort. Si tel est le cas, pourquoi le converti doit-il encore avoir peur de l'Enfer ? Car, pour Epicure, la mort est la privation totale de la sensibilité. Celui qui meurt n'a plus conscience de rien. Il ne connaît ni joie, ni peine, ni Enfer, ni Paradis. C'est pourquoi, dans sa Lettre à Ménécée sur la morale, Epicure nous apprend que personne ne peut expérimenter la mort. Tant que nous existons, elle ne nous concerne en rien et pendant qu'elle est là, nous n'existons plus.

La peur de l'Enfer est encore rejetée par Nietzsche qui refuse toute tentative de faire de Dieu un bourreau et de considérer l'Enfer comme le lieu du châtiment et de la faute. En battant en brèche la thèse de Schopenhauer selon laquelle le salut de l'homme consiste à s'affranchir du « vouloir vivre » et donc de la douleur qui en est l'expression, Nietzsche condamne aussi la morale du péché pour faire l'éloge de l'instinct. Pour lui, cette peur que prônent les religions conduit à la fuite de responsabilité face à l'existence. Cela se comprend dans la mesure où la religion relève de la «mentalité du troupeau »; puisqu'elle fait diminuer la force des instincts naturels qui sont refoulés dans l'inconscient et qui finalement se transforment en cruauté et en rancune souvent difficiles à oublier. Dans Généalogie de la morale, Nietzsche prend le converti qui a peur de l'Enfer et qui considère Dieu comme un

${ }^{8}$ Coran, Al-Madinah Al-Munawwarah, Complexe Roi Fahd pour l'impression du Noble Coran 1410,

$$
\text { S. } 2 \text {, V. } 81
$$


bourreau pour une bête triste et folle. Dans cet ordre d'idées, il refuse d'inventer une autre vie ; une vie différente de celle dans laquelle nous sommes. Dans Le gai savoir, il nous apprend que l'homme doit aimer la vie et œuvrer pour le bonheur. Il faut donc cesser d'être un fabricateur de dieu et de projeter en lui toutes ses intentions. Il doit plutôt devenir un «surhomme », c'est-à-dire un être supérieur, grâce à la «volonté de puissance ». La peur de l'Enfer ne stimule donc pas notre «volonté de puissance », mais la diminue. De ce point de vue, Nietzsche a voulu obliger ses lecteurs à s'interroger sur eux-mêmes et sur leur foi.

\section{B- Le désir de vengeance à l'origine de la conversion religieuse. Vengeance contre le diable:-}

En affirmant que Dieu existe, les hommes ne disent pas la même chose et ils ne sont pas nécessairement du même avis. En fait, le mot «Dieu» peut renvoyer à des idées très différentes. Chez certains convertis, Dieu peut être appelé au secours pour se venger contre le diable, parce qu'il est considéré comme un être tout puissant, c'est-à-dire, celui qui possède la force par lui-même et qui est capable de tout. C'est justement ce qui fait l'objet de notre réflexion dans la présente section.

En effet, les Saintes Ecritures nous enseignent que parmi les anges que Dieu a créés pour qu'ils soient près de lui et contemplent sa gloire, certains, à la suite du premier d'entre eux que l'on nomme le diable se sont rebellés contre lui. Cela signifie qu'ils ne peuvent plus rester près de Dieu et qu'ils sont rejetés dans l'Enfer préparé pour le diable et ses anges. C'est justement ce que nous apprend Jésus dans l'Evangile selon Saint Matthieu: "Alors il dira encore à ceux de gauche : allez loin de moi, maudits, dans le feu éternel qui a été préparé pour le diable et ses anges. ${ }^{9}$ Dès lors, les convertis pensent que le diable et ses anges viennent dans le monde des hommes pour apporter le mal ; qu'ils détournent les hommes de Dieu et les entraînent au péché. Sachant alors que le diable peut être la source du mal, mais qu'il n'est pas puissant et que seul Dieu est capable de tout, les personnes converties appellent Dieu au secours pour se venger contre le diable. Cela veut dire qu'en fait, les hommes seraient perdus si Dieu ne les aimait et ne les protégeait pas. D'ailleurs, selon la Sainte Bible, le diable est identifié comme le contradicteur de Dieu. Plusieurs noms lui sont attribués pour exprimer ses mauvaises actions. Selon les circonstances, il est appelé Satan ou Belzébuth. C'est le prince des ténèbres, le père du mensonge, le prince de ce monde.

Dans l'intention de nous montrer que seul, Dieu est capable de venger le converti contre le diable, l'apôtre Pierre, dans sa première épître, nous montre la nécessité de résister au diable par la foi en Dieu : "Soyez sobres, veillez. Votre partie adverse, le diable, comme un lion rugissant, rôde, cherchant qui dévorer. Résistez lui, fermes dans la foi. ${ }^{10}$ Alors, nous comprenons que Dieu n'abandonne pas le converti qui croit en lui. Il le garde, le gouverne avec sa divine providence et constitue en même temps celui qui le rend fort contre le diable et ses anges. Autrement dit, en Dieu, se trouve la confiance inébranlable qui lui permet de résister au diable ou de se venger contre lui.

Mais, à notre humble avis, une telle compréhension des Saintes Ecritures ne doit pas nous faire perdre de vue la réalité. L'homme peut lui-même choisir d'avoir le statut du diable, s'il décide de penser et d'agir contre la volonté de Dieu. Bien qu'étant converti à une religion, il est toujours libre. Par ses actes, il peut ressembler à Dieu ou au diable. S'il ressemble à Dieu, il écoutera sa parole et collaborera avec lui comme un fils bien aimé. S'il ressemble au diable, il deviendra son partenaire et fera le mal pour lui-même et pour autrui. Car, bien que la loi de Dieu soit exigeante, elle laisse néanmoins à chaque homme, la liberté de faire ce qu'il veut. Dans certaines circonstances, il peut faire ce qu'il veut, ce qui lui plait ou ce qu'il juge bon. Il peut dès lors ressembler à Dieu s'il veut ou ressembler au diable. Cela veut dire qu'en réalité, Dieu ne peut pas venger le converti contre le diable s'il décide lui-même, par ses actes, de ressembler au diable. Jean-Paul Sartre l'a bien compris quand il dit : "L'homme est responsable de ce qu'il est. ${ }^{11}$ Chaque converti, par ses actes, propose donc des valeurs. De ce point de vue, il peut prendre l'apparence de Dieu ou du diable, selon les circonstances. Cela veut dire qu'en réalité, le diable n'est pas nécessairement en dehors de l'homme. D'ailleurs, personne ne l'a jamais vu. C'est l'homme qui, selon les circonstances, prend l'apparence du diable par ses actes ou par ses comportements. Si les hommes pouvaient changer de mentalité et s'aimer entre eux, l'on ne parlerait plus de diable et l'on ne se vengerait plus contre lui.

\footnotetext{
${ }^{9}$ Mt 26, 41

${ }^{10}$ I P, 8-9

${ }^{11}$ Jean-Paul SARTRE, L'existentialisme est un humanisme, Paris, Nagel, 1970, p.24
} 


\section{Vengeance contre l'ennemi:-}

Il est clair que l'homme partage avec l'animal, l'instinct d'agressivité. C'est une tendance à déployer sa puissance qui peut impliquer la neutralisation de tout obstacle, y compris l'être humain, qui, d'une manière ou d'une autre, peut être considéré comme un ennemi. Dans ce sens, on comprend que l'agressivité est naturelle chez l'homme, quelle que soit la morale ou la religion et qu'il serait illusoire de la supprimer, mais de l'atténuer. C'est pourquoi le Coran recommande que la lutte soit engagée contre l'ennemi : "Combattez dans le sentier d'Allah ceux qui vous combattent, et ne transgressez pas. Certes, Allah n'aime pas les transgresseurs! ${ }^{12}$ D'ailleurs, philosophes et penseurs d'horizons les plus divers n'avaient pas attendu les travaux de Freud avant de montrer que la violence en général et le désir de vengeance contre l'ennemi, en particulier, est chez l'homme une tendance naturelle. Par exemple, dans Le citoyen ou les fondements de la politique, Thomas Hobbes nous apprend que l'homme porte en lui le désir de recourir aux moyens les plus extrêmes pour se venger contre son ennemi, en vue de sa propre conservation. De ce point de vue, il n'est pas étonnant que la religion soit pour le croyant un moyen pour se venger contre l'ennemi. Voilà ce que nous apprend Hobbes en ces termes : "Il s'en suit que, puisque chacun a droit de travailler à sa conservation, il a pareillement droit d'user de tous les moyens et de faire toutes les choses sans lesquelles il ne se pourrait point conserver. ${ }^{13}$

Partout et toujours, on voit donc l'homme exercer la violence contre ses semblables, en se vengeant contre ses ennemis. Cela veut dire qu'en ayant peur des phénomènes naturels comme nous l'avons montré dans la première section du chapitre précédent, les hommes ont encore peur d'eux-mêmes, quelle que soit leur confession religieuse. La crainte réciproque que les fidèles d'une même religion ou de religions différentes éprouvent vis-à-vis de l'autre, conditionne le rapport de chaque homme ou de chaque croyant envers son semblable et s'explique par le désir de vengeance contre l'ennemi. C'est pourquoi, dans Léviathan, Hobbes justifie l'existence d'une « crainte réciproque et une défiance universelle parmi les hommes. ${ }^{14}$ Même dans les religions, il n'est pas rare de voir des convertis agir pour détruire ou pour dominer leurs prochains, considérés comme leurs ennemis, afin que ceux-ci ne puissent en aucun cas empêcher la réalisation de leurs désirs.

S'inscrivant dans la même logique, Sigmund Freud a révélé, grâce à la psychanalyse (science de l'inconscient), l'existence de l'agressivité, une pulsion destructrice chez l'homme. A travers Malaise dans la civilisation, le père de la psychanalyse soutient : "L'homme est, en effet, tenté de satisfaire son besoin d'agression aux dépens de son prochain, d'exploiter son travail sans dédommagements, de l'utiliser sexuellement sans son contentement, de s'approprier ses biens, de l'humilier, de lui infliger des souffrances, de le martyriser et de le tuer. ${ }^{15}$

En parlant de la vengeance contre l'ennemi, nous pouvons encore nous référer à ce que nous dit René Girard dans La violence et le sacré. Pour cet auteur en effet, le sacré est l'organisation de la violence inhérente à la nature humaine. Autrement dit, loin de supprimer la violence primitive, le sacré la transforme en moyen de cohésion du groupe. Dans tous les cas, nous constatons que la violence et le sacrifice sont toujours mêlés dans l'acte fondateur des religions. Pour justifier sa thèse, Girard se fonde sur la notion de «victime émissaire » qui permet à une société ou à une religion de se décharger de son agressivité sur l'être toujours choisi comme un bouc émissaire que l'on sacralise afin de détourner la culpabilité provoquée par le meurtre rituel. Dès lors, nous pouvons parler de la religion en tant qu'un ensemble d'individus formant une communauté qui se réconcilie en Dieu ou en une autre divinité, aux dépens de la «victime émissaire». Pour René Girard, la violence est une caractéristique religieuse propre à toute société. Cela se traduit par la formule suivante : "Le religieux est tout entier orienté vers la paix mais les moyens de cette paix ne sont jamais dénués de violence sacrificielle. ${ }^{16}$

A analyser de près, nous ne pouvons pas ne pas donner raison à Hobbes, à Freud ou à Girard. Dans La philosophie bantoue, Placide Tempels a reconnu le totémisme comme un fondement de la religion chez les peuples primitifs. Une telle pratique met en relief l'idée du bouc émissaire ou de la victime émissaire dont nous parle René Girard dans La violence et le sacré. Dans le même ordre d'idées, nous pouvons justifier ces différentes thèses par ce que nous apprend Raoul Allier dans La psychologie de la conversion chez les peuples non-civilisés où il nous parle abondamment des pratiques relatives au sacrifice humain dans le paganisme. Ces pratiques étaient faites pour se

\footnotetext{
${ }^{12}$ Coran, op. cit, S. 2, V. 190

${ }^{13}$ Thomas HOBBES, Le citoyen ou les fondements de la politique, Paris, Ed Sirey, 1981, p. 84

${ }^{14}$ Thomas HOBBES, Léviathan, Paris, Ed Sirey, 1971, p.122

15 Sigmund FREUD, Malaise dans la civilisation, Paris, P.U.F., 1930 p.64

${ }^{16}$ René GIRARD, La violence et le sacré, Paris, Grasset, 1972, p. 41
} 
venger contre les ennemis et pour protéger les peuples afin de détourner d'eux, les fléaux. Marc Augé s'est lui aussi intéressé à la question de la vengeance contre l'ennemi quand il parle de « la guerre des frères ennemis à la mort du roi régnant. $\gg{ }^{17}$ Tous ces phénomènes nous donnent la preuve que la conversion religieuse relève de l'émotion et non de la raison.

Mais, loin d'encourager la violence à travers le fait religieux, nous voudrions par ces différentes considérations attirer l'attention de toute personne convertie sur la recherche d'une paix véritable à travers la conversion religieuse. Car, puisque Dieu est unique, tous les membres d'une même religion ou de différentes confessions religieuses doivent s'unir et s'aimer comme des frères pour faire échec à la violence ou du moins, pour l'atténuer.

Ainsi, dans la Catéchèse bantoue, un des premiers écrits pastoraux de Tempels, il a eu le mérite de souligner l'universalité du Christ. Pour l'auteur en effet, le Christ est venu sauver les hommes de toute race, de toute langue et de toute culture. Cela veut dire qu'en fait, le Christ n'est pas seulement venu pour la race blanche ou pour les peuples occidentaux. Que l'on soit un Africain ou un Européen, un Américain ou un Asiatique, on est appelé à aimer Dieu et son prochain comme soi-même pour bâtir un monde plus humain et mériter l'entrée au Paradis. A vrai dire, la foi en Dieu n'est pas une affaire de race, de culture de langue ou de classe sociale. Que l'on soit pauvre ou riche, jeune, adulte ou vieillard, on doit bien agir pour vivre en Dieu. Dans ce sens, le respect des commandements doit être une obligation et une réalité, conformément à la logique de Tempels. Dans le même ordre d'idées, il faut dire que l'appartenance à une religion ou à une autre ne garantit pas le salut de Dieu. Ce qui importe, c'est d'accomplir sa volonté. Dans ce sens, nous disons ici et maintenant que la guerre des religions n'est qu'une barbarie ; puisqu'en réalité, nul ne peut se servir de la violence pour justifier la foi. Le Dalaï Lama a donc raison quand il affirme qu'il croit fermement aux valeurs de la non-violence.

\section{Vengeance contre le plus fort:-}

La présente section nous oriente vers la question du plus fort ; une question qui, depuis l'Antiquité Grecque, est au centre de la réflexion philosophique en général et de la philosophie morale et politique en particulier. En effet, le plus fort désigne un individu qui possède une puissance d'action physique ou symbolique résultant d'une pluralité de sources. Chez le plus fort, la force peut se manifester par des techniques d'exploitation des plus faibles à travers l'agression physique ou la mise en œuvre des moyens rationnels pouvant permettre la destruction d'un homme ou d'un groupe d'hommes. Autrement dit, le plus fort a le pouvoir d'exercer une action sur quelqu'un pour l'amener à faire quelque chose, indépendamment de sa volonté. Depuis Calliclès, il est clair que le plus fort attaque le plus faible pour avoir tous les droits. D'ailleurs, l'histoire nous révèle que le droit du plus fort est le plus constant de tous les droits. Dans Le loup et l'agneau, La Fontaine souligne que le plus fort a toujours raison, en ce sens qu'il a le pouvoir d'exploiter le plus faible et d'obtenir de lui tout ce qu'il veut, quand il veut et où il veut.

Par contre, dans Du contrat social, Jean-Jacques Rousseau a mis en relief la contradiction inhérente au prétendu droit du plus fort. Pour Rousseau en effet, la force ne doit en aucun cas faire le droit. Car, selon les circonstances, elle peut s'affaiblir pour faire place à une autre force supérieure. Nous pouvons nous référer à la formule suivante : «Le plus fort n'est jamais assez fort pour être toujours le maître. ${ }^{18}$ Aussi longtemps qu'un individu est le plus fort, c'est cette force qui est la source de son pouvoir. Si quelqu'un lui obéit, c'est parce qu'il est plus faible que lui et qu'il le craint, en ce sens qu'il ne peut faire autrement. Dieu est l'être puissant qui a la force par lui-même. C'est encore lui qui donne sa force à celui qui en a et qui domine les autres. Mais, pour l'auteur de : Du contrat social, la force peut prendre fin et redevenir faible en face d'une force supérieure. Car, seul Dieu est le plus fort ; tandis que la force humaine est limitée.

Dans cet ordre d'idées, nous pouvons comprendre comment la religion peut permettre aux plus faibles de se venger contre le plus fort. Dans la première partie de notre thèse, nous avons défini la religion, conformément à la formule de Durkheim. Dans la religion, les plus faibles se mettent ensemble pour former une communauté qui s'enracine dans une histoire commune et qui repose sur un patrimoine commun. De ce point de vue, toute religion engendre

\footnotetext{
${ }^{17}$ Marc AUGE, Pouvoirs de vie, pouvoirs de mort : introduction à une anthropologie de la répression, Paris, Flammarion, 1977, p. 129

18 Jean-Jacques ROUSSEAU, Du contrat social, Paris, Garnier-Flammarion, 2001, p.44
} 
entre ses membres ou entre ses adeptes, un sentiment naturel de solidarité. Il s'agit d'une solidarité à travers laquelle les faibles se réunissent ou s'assemblent pour se venger contre le plus fort.

Dans la religion, l'intérêt particulier et l'intérêt général semblent être confondus. En tout cas, on constate que leur rapport est immédiatement senti. Quiconque attaque un membre a affaire à toute la communauté. Dans ce sens, nous pouvons paraphraser Rousseau pour dire que la religion constitue une forme d'association dont le but est de défendre et de protéger la personne et les biens de chaque associé. Car, les membres d'une religion se considèrent le plus souvent comme des fils et filles d'un même père. C'est pourquoi, ils se vengent contre leurs ennemis en croyant puiser dans la force de Dieu pour agir. Dieu a un pouvoir qui permet à ses fidèles de briser toutes les résistances que l'ennemi leur oppose. Il est lui-même la force suprême à laquelle aucune autre ne peut résister.

\section{Vengeance contre l'incrédule:-}

Dans l'imaginaire collectif, tout se passe comme si les convertis ont un important compte en banque, en ce sens qu'ils se croient plus aimés de Dieu par rapport à l'incrédule. Autrement dit, la foi en Dieu apparaît comme un synonyme de garantie ou d'assurance. Elle est la manifestation de la confiance absolue en Dieu, une confiance fondée sur des témoignages dont la raison ne saurait totalement garantir la valeur. Ainsi, pour Gérard Durozoï et André Roussel, «la foi désigne alors une croyance ferme, une certitude qui ne s'appuie pas sur des preuves rationnelles. $\gg{ }^{19}$ En d'autres termes, elle est l'adhésion aux dogmes qui sont considérés comme des vérités révélées.

Toutefois, on note chez le converti un certain sentiment de vengeance à l'égard de l'incrédule, c'est-à-dire, à l'égard de celui qui ne croit pas en Dieu. Pourtant, à travers les Saintes Ecritures, on constate que Dieu s'est révélé aux hommes de toute race, de toute culture et de toute langue, de manière progressive et pédagogique. S'il est vrai qu'au départ, il s'est choisi et façonné un petit groupe, c'est-à-dire le peuple d'Israël, pour contracter une alliance avec lui, il faut néanmoins reconnaître qu'à travers ce peuple, ce sont en même temps tous les peuples de la terre qui doivent bénéficier de son salut. Autrement dit, Dieu n'accorde pas seulement ses bienfaits à ceux qui appartiennent à une religion particulière, mais à tous les hommes. Que l'on soit un fidèle ou un adepte d'une religion ou non, il est avec nous et exauce nos prières comme celles des autres. Cela veut dire qu'en réalité, le salut de Dieu s'étend à toute l'humanité. L'essentiel n'est pas d'appartenir à une religion pour prétendre être sauvé. Pour notre part, ce qui est important dans la question du salut de Dieu, c'est de bien penser pour bien agir, conformément aux lois morales de la société ; une société qui tient son existence de Dieu.

Si vraiment l'individu s'évertue à respecter avec une bonne conscience les lois justes de la société, il est déjà en accord avec Dieu et il peut jouir de son salut, au même titre que le fidèle ou l'adepte d'une religion. Cela veut dire qu'en réalité, il y a un rapport très étroit entre les commandements de Dieu et les lois de la société. Voilà ce que nous apprend Durkheim dans ce sens : "D’une manière générale, il n'est pas douteux qu'une société a tout ce qu'il faut pour éveiller dans les esprits, par la seule action qu'elle exerce sur eux, la sensation du divin; car elle est à ses membres ce qu'un Dieu est à ses fidèles. ${ }^{20}$ De ce point de vue, nous comprenons dès lors que la société revêt un pouvoir important. On pourrait même dire qu'il s'agit d'un pouvoir qui vient de Dieu, en ce sens qu'elle nous aide à prendre conscience de nous-mêmes et des règles morales adéquates à respecter pour être de bons citoyens. Or, à dire toute pensée, le bon citoyen est déjà un bon converti ; tandis que le mauvais citoyen n'est qu'un mauvais converti. Car, l'influence que la société exerce sur nos consciences tient beaucoup de l'autorité morale dont elle est investie. En obéissant aux lois de la société par l'accomplissement de nos devoirs, nous aimons déjà le prochain et nous aimons déjà Dieu. Ainsi, nous ne sommes plus des incrédules, mais plutôt des vrais convertis. Pour ceux qui nous reprochent d'égaler Dieu à la société, nous contestons par l'affirmation suivante : Nous ne posons pas l'équation selon laquelle la société représente Dieu ou que Dieu est égal à la société. Au contraire, nous invitons les hommes de toute race, de toute culture et de toute langue à mieux connaître Dieu pour mieux l'adorer.

${ }^{19}$ Gérard DUROZOI - André ROUSSEL, Dictionnaire de philosophie, Paris, Nathan, 2002, p.153

${ }^{20}$ Emile DURKHEIM, Les formes élémentaires de la vie religieuse : le système totémique en Australie, Paris, P.U.F., 1998, p.295 


\section{Vengeance contre le pécheur:-}

Chez les personnes converties, l'émotion consiste encore à se venger contre les pécheurs, c'est-à-dire, ceux qui font du mal et qui refusent de se convertir. Pourtant, dans l'Evangile, saint Jean nous parle des Scribes et des Pharisiens qui amènent une femme adultère à Jésus en lui disant: "Maître, cette femme a été surprise en flagrant délit d'adultère. Or, dans la loi, Moïse nous a prescrit de lapider ces femmes-là. Toi donc, que dis tu? $\gg^{21}$ Puisque ces Scribes et Pharisiens persistaient pour connaitre la réponse de Jésus sur cette question, il leur a répondu en ces termes: "Que celui d'entre vous qui est sans péché lui jette le premier une pierre! » ${ }^{22}$ Après avoir entendu et compris la réponse de Jésus, les accusateurs s'en allaient, l'un après l'autre, à commencer par les plus anciens. S'étant finalement retrouvé seul avec la femme, Jésus lui demande où sont passés ses accusateurs. Ensuite, il lui demande si personne ne l'avait condamnée. Alors, la femme répond en disant que personne parmi ses accusateurs ne l'avait condamnée. C'est alors que Jésus lui dit : «Moi non plus, je ne te condamne pas. Vas, désormais ne pêche plus. $»^{23}$

Cette rencontre de Jésus avec la femme adultère nous donne la preuve que Dieu ne veut pas que l'on se venge contre le pécheur et qu'il ne veut pas non plus sa mort. A vrai dire, la souffrance éternelle est quelque chose que personne ne doit jamais souhaiter, même à son pire ennemi. C'est un malheur inimaginable qui, en réalité, ne doit pas être pensé, quelles que soient les circonstances. Même si le fidèle sent un désir de vengeance dans les propos des chefs religieux (prêtres, pasteurs, imams ou chefs religieux traditionnels), il doit lui-même se poser des questions. Car, tant que l'on arrive à croire simplement à ce que dit son chef religieux sans se poser de question, tout va bien. Mais lorsqu'on commence à réfléchir par soi-même, surtout au sujet de la vengeance contre l'ennemi ou contre le pécheur, on a de la matière à passer des nuits blanches. Jésus ne condamne pas et n'évite pas non plus le pécheur. Les Saintes Ecritures révèlent qu'il va même au-devant d'eux pour les amener à la conversion. Plusieurs fois, il a fréquenté les pécheurs et a mangé avec eux. L'exemple de Matthieu le Publicain, l'illustre mieux. Mais, reconnaissons que Jésus ne cautionne pas le péché. Il veut plutôt que le pécheur se convertisse pour devenir un homme nouveau.

En d'autres termes, Dieu n'enfonce jamais le clou de la culpabilité. Par sa miséricorde, il soulage de leur fardeau, ceux que le poids du péché a fait tomber à terre, afin qu'ils se redressent pour continuer à vivre. Autrement dit, Dieu ne veut pas que le pécheur soit puni, mais qu'il revive tout en reconnaissant son œuvre dans sa vie. A notre avis, si nous évitons de nous venger contre le pécheur en lui montrant ce qu'il doit faire et comment y parvenir, il mènera une vie plus juste. Car la bonté et le pardon de Dieu sont sans limite. Soulignons que la vengeance contre le pécheur ne peut en aucun cas permettre au converti d'avoir la paix. Comme nous l'apprend saint Augustin, pour avoir la paix, il faut aimer. D'ailleurs, il serait impossible d'identifier au sein de l'humanité, les pécheurs de ceux qui ne pêchent pas. Alain a donc raison quand il dit que, ce sont les mêmes hommes qui font la guerre qui aiment encore la paix. Pour notre part, il vaut mieux travailler pour sa propre sainteté et contribuer à celle des autres que de penser à l'existence d'un Dieu vengeur qui doit torturer dans l'Enfer, ceux qui pèchent ici-bas.

\section{Conclusion:-}

La promotion de la paix au sein des religions doit nécessairement passer par la maîtrise des émotions chez les personnes converties. On sait qu'il y a des émotions nocives, c'est-à-dire négatives et des émotions positives. En fait, la religion devrait délivrer le converti des émotions négatives telles que la peur et le désir de vengeance. Elle devrait donner au converti, la joie de se sentir membre d'une communauté de fidèles qui doivent véritablement aimer Dieu et leurs prochains comme eux-mêmes. Puisque l'homme est un être ambivalent, c'est-à-dire capable du meilleur et du pire, la paix doit dépendre de l'homme véritablement transformé par la Parole de Dieu ; un homme prêt à lutter contre la peur et le désir de vengeance pour aimer ses semblables. En réalité, il serait illusoire de croire que, pour être un bon converti, on doit devenir l'ennemi des incrédules ou des fidèles d'autres religions. Etre un bon converti, c'est lutter contre la peur et le désir de vengeance en souhaitant par la raison le bien pour autrui et pour soi-même. Si tel n'est pas le cas, le converti doit se poser des questions sur sa foi. Car, bien que le rôle de la raison dans la conversion religieuse soit limité, elle doit néanmoins permettre de distinguer le vrai du faux, voire le bien du mal. Autrement dit, la raison doit permettre au converti de savoir s'il est en accord ou en désaccord avec la Parole de Dieu.

\section{${ }^{21} \mathrm{Jn}, 8,4$}

${ }^{22} \mathrm{Jn}, 8,7$

${ }^{23} \mathrm{Jn}, 8,11$ 


\section{Bibliographie:-}

1. ALLIER R., La psychologie de la conversion chez les peuples non-civilisés, Paris, Payot, 1925

2. AUGE M., Pouvoirs de vie, pouvoirs de mort : introduction à une anthropologie de la répression, Paris, Flammarion, 1977

3. CORAN, Al-Madinah Al-Munawwarah, Complexe Roi Fahd pour l'impression du Noble Coran, 1410.

4. DALAÏ LAMA, Au-delà des dogmes, Paris, Albin Michel, 1994

5. DURKHEIM E., Les formes élémentaires de la vie religieuse : le système totémique en Australie, Paris, P.U.F., 1968

6. DUROZOI G.- ROUSSEL A., Dictionnaire de philosophie, Paris, Nathan, 2002

7. EPICURE, Lettre à Ménécée sur la morale, Paris, Hatier, 1984

8. FEUERBACH L., Principes de la philosophie de l'avenir, Paris, P.U.F., 1843

9. FREUD S., Malaise dans la civilisation, Paris, P.U.F., 1930

10. FREUD S., Essai de psychanalyse, Paris, Payot, 1972

11. GIRARD R., La violence et le sacré, Paris, Grasset, 1972

12. HEBGA M., Sorcellerie et prière de délivrance : réflexion sur une expérience, Paris, Présence Africaine, 1982

13. HEIDEGGER M., Introduction à la métaphysique, Paris, Gallimard, 1953

14. HOBBES T., Léviathan, Paris, Ed Sirey, 1971

15. HOBBES T., Le citoyen ou les fondements de la politique, Paris, Ed Sirey, 1981

16. HOUNTONDJI P., Sur la philosophie africaine : critique de l'ethnophilosophie, Paris, Maspero, 1977

17. JACOB F., La logique du vivant, Paris, Gallimard, 1978

18. LA BIBLE DE JERUSALEM, Paris, Cerf, 2001

19. LEIBNIZ G.-W., Principes de la nature et de la grâce fondés en raison, Paris, P.U.F., 1986

20. LUCRECE, De la nature, Paris, Les Belles Lettres, 1924

21. NIETZSCHE F., Le gai savoir, Paris, Gallimard, 1972

22. NIETZSCHE F., La généalogie de la morale, Paris, Nathan, 1981

23. ORTIGUES M.-C - E., Edipe africain, Paris, L'Harmattan, 1966

24. ORTIGUES E., Religions du livre et religions de la coutume, Paris, Les éditions le Sycomore, 1981

25. PASCAL B., Pensées, Paris, Gallimard, 1976

26. PLATON, Gorgias, Paris, Garnier-Flammarion, 1967

27. PLATON, Gorgias, Paris, Garnier-Flammarion, 1967

28. ROUSSEAU J.-J., Du contrat social, Paris, Garnier-Flammarion, 2001

29. SARTRE J.-P., L'existentialisme est un humanisme, Paris, Nagel, 1970

30. SCHOPENHAUER A., Le monde comme volonté et comme représentation,Paris, P.U.F., 1966

31. TEMPELS P., La philosophie bantoue, Elisabethville, Lovania, 1945 